\title{
Special announcement: Guidelines to the Practice of Anesthesia - Revised Edition 2018
}

\author{
Gregory Dobson, MD
}

Received: 12 September 2017/ Accepted: 14 October 2017/Published online: 3 November 2017

(c) Canadian Anesthesiologists' Society 2017

This issue of the Journal features the 2018 Revised Edition of the Guidelines to the Practice of Anesthesia ${ }^{1}$ as developed and annually updated by the Canadian Anesthesiologists Society (CAS) Committee on Standards and approved by the CAS Board of Directors. The CAS has endorsed the Guidelines since their initial development in 1974 (and publication in 1977). The Journal has published yearly updates since 2009. The Guidelines are widely recognized as a valuable clinical resource for anesthesia practitioners both nationally and internationally. Indeed, the Guidelines are frequently downloaded as free web content and regularly cited in other peer-reviewed articles.

The Committee on Standards welcomes questions, comments, and constructive criticism. Any queries and the related responses are available on the CAS website. Indeed, this editorial commentary should be considered an invitation to any anesthesiologist, allied health professional, or healthcare administrator to forward questions or comments to the Committee on Standards (standards@cas.ca).

In view of the comprehensive and lengthy nature of the Guidelines, this editorial is meant to draw readers' attention to specific topics updated in the new version. It is also a commentary on other issues undergoing further consideration for publication in future versions of the Guidelines.

Readers will notice a new number-indexed referencing system in the Table of Contents and in the text of the document designed to make it easier to reference specific

\section{G. Dobson, MD ( $\square)$}

Committee on Standards, Canadian Anesthesiologists' Society,

1 Eglinton Avenue East, Suite 208, Toronto, ON M4P 3A1,

Canada

e-mail: G.Dobson@Dal.Ca sections and subsections of the Guidelines. Some new subsection headings have also been created. Readers who are familiar with earlier editions of the Guidelines may also notice that the 2018 edition has a significantly updated and improved new look. In addition to the material (i.e., content changes) in the Guidelines, some of the language in the document has been revised to improve accuracy, readability, and fitness to its purpose, as well as to be sure it is free from errors, omissions, inconsistencies, and/ or repetitions. Great care has been taken to avoid unintentional alterations in the content, context, or meaning of material when compared to earlier editions. We sincerely hope and anticipate that readers will agree that it is a much improved document. As appendices to the Guidelines are revised and updated in the future, they will be subjected to similar revision. Of note, we are currently busy updating and revising Appendix 6: Position Paper on Procedural Sedation. ${ }^{2}$ We are looking forward to publishing the improved document with a future edition of the Guidelines.

The Committee actively considered a variety of topics for revision and implemented numerous changes, which are highlighted in the revised edition of the Guidelines. The following changes are among those implemented, appearing in italics below.

The Guidelines currently acknowledge the responsibility of the Chief of Anesthesia to monitor systematically the quality of anesthetic care provided throughout the healthcare facility. Ever-increasing expectations are being placed on clinical departments by health facilities and health ministries to develop and implement robust quality improvement programs. The Committee has responded to these expectations with additional content in the 2018 Revised Edition. It is important to emphasize that the extent of quality improvement activities will vary widely 
depending on the resources available to the Chief of Anesthesia. It is a responsibility of the Chief of Anesthesia "To employ a systematic approach for monitoring the quality of anesthetic care provided by members of the department of anesthesia throughout the healthcare facility. Monitoring quality of care may include, but need not be restricted to, the use of chart audits, clinical indicator and outcome monitoring, adverse event reporting systems, morbidity and mortality conferences and critical incident case reviews. The extent of quality improvement activities will vary depending on the departmental and health facility resources available to the chief. For effective quality improvement it is strongly encouraged that all department members should actively participate in any such activities."

Cognitive Aids in the context of healthcare are physical or virtual items to help professionals execute the complex decision making of diagnosis and therapy in a timely fashion. They function as prompts to assist providers to complete a series of tasks, such as during a critical emergency. Well-designed cognitive aids together with team education and simulation may improve performance, response times, and outcomes during critical anesthetic emergencies. ${ }^{3}$ Therefore, the Guidelines contain new material in section 3.0 - Anesthesia Equipment and Anesthetizing Location - and recommend their use, stating, "It is strongly recommended that a cognitive aid manual be made available at all anesthetizing locations in support of the management of critical perioperative emergencies. The contents of the manual should be regularly reviewed and updated."

The Committee annually reviews the recommendations for preoperative testing and revises them periodically as required based on the latest available recommendations, such as Choosing Wisely Canada., ${ }^{4,5}$ The Committee has chosen to add more specific recommendations for preoperative testing in asymptomatic patients undergoing low-risk surgery. While acknowledging that the definition of low-risk surgery has been the subject of some discussion and debate, the Committee recommends that "Routine laboratory blood testing, electrocardiograms and chest radiographs are not recommended for asymptomatic patients having low risk surgery. Examples of low risk surgery include, but are not restricted to, cataract surgery, minor breast surgery, superficial procedures and many ambulatory procedures. For more detailed definitions of low risk surgery and for other recommendations related to preoperative testing visit Choosing Wisely on the CAS website 4 and the associated internet links." This edition of the Guidelines also contains entirely new indications for preoperative electrocardiograms and chest radiographs for patients undergoing surgery considered not to be low-risk procedures. The Committee chose to include the distinction "intermediate-risk" surgery in addition to "high-risk" surgery for electrocardiograms, recognizing that there are several surgical risk stratification calculators presently available for clinical use. The blood test section of the Guidelines' test table is currently under review, and readers can expect to see updated recommendations in the Revised Edition 2019. The Committee is actively discussing the possibility of adding specific recommendations to future editions for other preoperative tests, such as echocardiography, exercise, and radionucleotide stress testing, as well as other preoperative investigations for patients with known or suspected heart disease while recognizing that guidelines for these tests are currently available in the literature and indeed vary in their recommendations.

The critical importance of maintaining perioperative normothermia (in the absence of specific indications for hypothermia) has been well established. Potential complications of hypothermia include wound infection, major adverse cardiac events, immune dysfunction, coagulopathy, increased blood loss, and transfusion requirements. The Committee has chosen to add recommendations for perioperative core temperature management. They appear in two sections of the revised edition. In subsection 5.1 - Preparation for Anesthesia readers will find the recommendation that required equipment should be available and in working order, “...including the equipment required for supporting core temperature management (patient core temperature 36$37^{\circ} \mathrm{C}$ )." In addition, a new subsection - 5.5 Perioperative Temperature Management - has been created that contains the statement that "Monitoring patient core temperature is strongly recommended during cases of general and neuraxial regional anesthesia lasting 30 minutes or longer. In the absence of surgical or patient indications for intraoperative hypothermia, active patient warming systems, control of the operating room ambient temperature and other methods should be used to target a central core temperature of $36-37^{\circ} \mathrm{C}$."

Patient handover from one care provider or team to another has been well recognized as a vulnerable time relative to the potential for failure to communicate key information pertinent to patient care and the increased risk for adverse events and adverse outcomes. As such, the Committee added recommendations for patient care handovers in the operating room and for transfer of accountability in the post-anesthesia care unit (PACU). Transfer of care in other situations (e.g., labour and delivery unit, emergency department, intensive care unit) are not specifically addressed in the Guidelines, but the same principles should apply during any patient handover scenario. The Guidelines now state, "An intraoperative handover of care between two anesthesiologists should be 
documented in the anesthesia record and follow a structured protocol" Communication of necessary information to PACU staff should be completed "as part of a structured handover of care protocol."

The Guidelines have been silent to date regarding recommendations for the administration and for monitoring neuromuscular blocking agents. The Committee strongly supports optimizing patient safety and therefore attention to detail with respect to the use of non-depolarizing neuromuscular blocking agents. This is now addressed in subsection 5.3 - Patient Monitoring - which states, "Cautious dosing, vigilant monitoring and the appropriate reversal of neuromuscular drugs are all essential for patient safety. Neuromuscular monitoring should be utilized when non-depolarizing neuromuscular blocking agents are administered."

Anesthesia care during patient transport can represent a period of increased risk due to an interruption in continuous monitoring. As such, the need for specific transport monitoring should be determined by the condition and level of consciousness of the patient and the potential risk for hypoxemia and respiratory depression. Appropriate monitoring devices should be utilized when indicated, "such as, but not restricted to, portable pulse oximetry." Capnography is required for intubated or deeply sedated (RSS 4-6) patients during their stay in the PACU. This statement was added to the Guidelines in the Revised Edition 2016. ${ }^{6}$ Although capnography for patient transport is not specifically mentioned in the Guidelines, it should be considered, if available, during transport of patients who are intubated. Occasionally patients who have a difficult airway require reintubation in the PACU. In some facilities, the PACU may be located some distance from the operating rooms, and thus it is not always be possible to transport these patients to the operating room for airway management. Hence, "Equipment for management of the difficult airway must be immediately available to the PACU."

Other topics the Standards Committee is actively discussing for consideration in future editions of the Guidelines include acute pain management guidelines (e.g., ketamine infusions), retirement and pre-retirement "winding down" recommendations, and guidelines specific to pediatric anesthesia. The Committee on Standards continues to work in cooperation with the Committee on the Anesthesia Care Team (COACT) to support the continued development of the anesthesia assistant's role and the practice guidelines reflected in Appendix 5: Position Paper on Anesthesia Assistant. ${ }^{7}$ We, the CAS Committee on Standards, welcome suggestions for other issues and topics that readers believe should be addressed in the Guidelines.

In conclusion, I wish to acknowledge the passing of one of our own and someone who played a very important part in the history of our Guidelines. Those who read the editorial $^{8}$ that accompanied the Revised Edition 2017, and in fact many other Canadian anesthesiologists, know that Dr. John Feindel created the very first version of our Guidelines, published in 1977. After an illustrious career and a long retirement, he passed away in July 2017, at the age of 86. The Committee on Standards honour him and his vision, and we offer our heartfelt condolences to his family. His legacy will live on in our Guidelines.

\section{Annonce spéciale : Guide d'exercice de l'anesthésie - Édition révisée 2018}

Ce numéro du Journal présente l'édition révisée du Guide d'exercice de l'anesthésie ${ }^{1} 2018$, tel qu'il a été mis au point et annuellement mis à jour par le Comité des normes de la Société canadienne des anesthésiologistes (SCA), puis approuvé par le Conseil d'administration de la SCA. La SCA appuie le Guide depuis sa première mise au point, en 1974 (et sa publication en 1977). Le Journal publie des mises à jour annuelles depuis 2009. Le Guide est reconnu en tant que ressource clinique inestimable, tant par les fournisseurs de soins anesthésiques au pays qu'à l'échelle internationale. En effet, le Guide est fréquemment téléchargé sous forme de contenu web gratuit et régulièrement cité dans d'autres articles révisés par les pairs.

Le Comité des normes reçoit volontiers toute question, tout commentaire, et toute critique constructive. Toutes les questions et leurs réponses sont disponibles sur le site Internet de la SCA. En effet, ce commentaire éditorial constitue une invitation aux anesthésiologistes, professionnels paramédicaux ou administrateurs de services de santé, à faire parvenir toute question et tout commentaire au Comité des normes (standards@cas.ca).

$\mathrm{Au}$ vu de la nature exhaustive et relativement longue du Guide, cet éditorial a pour but d'attirer l'attention du lecteur vers des sujets en particulier qui ont été mis à jour dans cette version révisée. Il constitue également un commentaire concernant d'autres questions et sujets qui sont présentement soumis à un examen plus minutieux avant d'être publiés dans une version future de ce Guide.

Le lecteur notera le nouveau système de référencement numéroté dans la table des matières et dans le corps du texte. Cette modification a pour but de faciliter le référencement à des sections et sous-sections spécifiques du Guide. De nouvelles entêtes de sous-section ont également été ajoutées. Le lecteur familier avec les anciennes versions du Guide remarquera également que 
l'édition 2018 jouit d'une nouvelle apparence revue au goût du jour et améliorée. Outre les révisions d'ordre 'matériel' (c'est-à-dire, des modifications du contenu) au Guide, le texte utilisé dans le document a aussi été passé en revue afin d'améliorer la précision, la lisibilité et l'aptitude du Guide à remplir son rôle, ainsi que pour s'assurer qu'il ne contient ni erreurs, omissions, incohérences, ou répétitions. Au cours de ce processus de révision, nous avons porté une attention particulière à éviter toute altération involontaire au contenu, au contexte ou à la signification du matériel par rapport aux éditions précédentes. Nous espérons et prévoyons en toute sincérité que le lecteur trouvera lui aussi ce document considérablement amélioré. Au fur et à mesure que les Annexes au Guide seront révisées et mises à jour à l'avenir, elles seront sujettes à de semblables révisions. Il est à noter que nous sommes en train de mettre à jour et de réviser l'Annexe 6: Exposé de principe sur la sédation consciente. $^{2}$ Nous envisageons de publier ce document amélioré avec une version future du Guide.

Le Comité a examiné en détail plusieurs sujets pour les réviser et a intégré de nombreuses modifications, lesquelles sont surlignées dans la version révisée du Guide. Les modifications suivantes, mises en exergue en italique ci-dessous, en sont quelques exemples.

Le Guide reconnaît actuellement la responsabilité du chef du département d'anesthésie dans la surveillance systématique de la qualité des soins anesthésiques offerts à la grandeur de l'établissement de soins de santé. Des attentes toujours plus élevées sont placées sur les départements cliniques par les établissements de santé et les ministères de la santé afin qu'ils mettent au point et implantent de solides programmes d'amélioration de la qualité. Le Comité répond à ces attentes en ajoutant du contenu dans l'édition 2018. Il est important de souligner que l'étendue des activités d'amélioration de la qualité variera beaucoup en fonction des ressources mises à la disposition du chef du département d'anesthésie. L'une des responsabilités du chef du département d'anesthésie sera de : "Avoir recours à une approche systématique pour surveiller la qualité des soins anesthésiques offerts par les membres du département d'anesthésie à la grandeur de l'établissement de soins de santé. La surveillance de la qualité des soins peut comprendre, sans cependant s'y limiter, le recours à des vérifications de dossiers, la surveillance d'indicateurs et de suivis cliniques, les systèmes de surveillance des évènements indésirables, des conférences sur la morbidité et la mortalité, et la révision des cas d'incidents critiques. L'étendue des activités d'amélioration de la qualité variera selon les ressources départementales et institutionnelles mises à la disposition du chef de département. Pour une amélioration efficace de la qualité, tous les membres du département sont fortement encouragés à prendre une part active à de telles activités. »

Dans le contexte des soins de santé, les listes de contrôle ou aides cognitives sont des items physiques ou virtuels dont l'objectif est d'aider les professionnels à prendre les décisions complexes que constituent le diagnostic et le traitement dans un délai adapté. Ces listes sont des outils destinés à aider les fournisseurs de soins à compléter une série de tâches, comme par exemple pendant une urgence critique. Les listes de contrôle et aides cognitives bien conçues, lorsqu'elles s'accompagnent de formation et de simulation pour l'équipe, peuvent améliorer la performance, les délais de réponse et les pronostics en cas d'urgence anesthésique critique. ${ }^{3}$ Par conséquent, le Guide comprend du nouveau contenu dans la section 3.0 Matériel d'anesthésie et lieux convenant à l'anesthésie - et recommande l'utilisation de telles listes et aides cognitives, déclarant, "Il est fortement recommandé qu'un manuel de listes de contrôle et d'aides cognitives soit disponible dans tous les lieux où l'anesthésie est pratiquée afin de soutenir la prise en charge des urgences périopératoires critiques. Le contenu de ce manuel devrait être révisé et mis à jour régulièrement. »

Chaque année, le Comité passe en revue les recommandations concernant les examens préopératoires et les révise périodiquement si nécessaire, en fonction des recommandations les plus récentes, telles que Choisir avec soin Canada. ${ }^{4,5}$ Le Comité a choisi d'ajouter des recommandations plus spécifiques concernant les examens préopératoires chez les patients asymptomatiques subissant une chirurgie à faible risque. Tout en étant conscient que la définition d'une chirurgie à faible risque fait l'objet de discussion et de débat, selon le Comité, "Les tests sanguins de laboratoire, les électrocardiogrammes et les radiographies $d u$ poumon de routine ne sont pas recommandés chez les patients asymptomatiques subissant une chirurgie à risque faible. Les chirurgies de cataracte, les chirurgies mammaires mineures, les interventions superficielles et bon nombre d'interventions ambulatoires mineures sont quelques exemples de chirurgies à risque faible. Pour obtenir une définition plus détaillée des chirurgies à faible risque et d'autres recommandations concernant les examens préopératoires, consultez la section Choisir avec soin du site de la SCA ${ }^{4}$ et les liens internet associés. »Cette édition du Guide comprend également de toutes nouvelles indications concernant les électrocardiogrammes préopératoires et les radiographies du poumon pour les patients devant subir une chirurgie qui n'est pas considérée comme une intervention à faible risque. Le Comité a décidé d'inclure la distinction de chirurgie «à risque intermédiaire » en plus de la chirurgie «à risque élevé » pour les électrocardiogrammes. Ainsi, il reconnait qu'il existe de nombreux outils de stratification du risque 
chirurgical destinés à un usage clinique. La section des tests sanguins dans le tableau des examens du Guide est présentement en révision, et le lecteur pourra voir des recommandations adaptées dans l'édition révisée de 2019. Le Comité est en discussion concernant la possibilité d'ajouter des recommandations spécifiques dans les éditions futures portant sur d'autres examens préopératoires tels que l'échocardiographie, l'exercice et le test de stress en médecine nucléaire, ainsi que d'autres examens préopératoires pour les patients présentant une cardiopathie connue ou soupçonnée, tout en étant conscient qu'il existe des lignes directrices concernant la pertinence de ces tests dans la littérature et qu'elles varient dans leurs recommandations.

L'importance cruciale du maintien de la normothermie périopératoire (en l'absence d'indications spécifiques justifiant une hypothermie) a été bien établie. Parmi les complications potentielles de l'hypothermie, citons les infections, des complications cardiaques majeures, un dysfonctionnement du système immunitaire, une coagulopathie, une augmentation de la perte de sang, et des besoins transfusionnels. Le Comité a décidé d'ajouter des recommandations concernant la prise en charge périopératoire de la température centrale. Ces recommandations apparaissent dans deux sections de l'édition révisée. Dans la sous-section 5.1 - Préparation à l'anesthésie - le lecteur retrouvera la recommandation selon laquelle le matériel requis devrait être accessible et en bon état de fonctionnement, "y compris le matériel nécessaire au maintien de la température centrale (température centrale du patient $36-37^{\circ} \mathrm{C}$ ). » En outre, une nouvelle sous-section - 5.5. Prise en charge périopératoire de la température - a été créée et comporte l'énoncé suivant : «Le monitorage de la température centrale du patient est fortement recommandé pendant les cas d'anesthésie générale et d'anesthésie régionale neuraxiale d'une durée de 30 min ou plus. En l'absence d'indications chirurgicales ou liées au patient concernant une hypothermie peropératoire, des systèmes de réchauffement du patient actifs, le contrôle de la température ambiante en salle d'opération et d'autres méthodes devraient être utilisés pour cibler une température centrale de $36-37^{\circ} \mathrm{C}$. »

Il a été clairement établi que le transfert d'un médecin traitant ou d'une équipe de soins à une autre est une période vulnérable en raison du risque de non-communication d'informations clés pertinentes aux soins du patient et du risque accru d'événements indésirables et de pronostics indésirables. C'est pourquoi le Comité a ajouté des recommandations concernant le transfert des soins du patient en salle d'opération et le transfert de l'imputabilité à la salle de réveil. Le transfert des soins effectué dans d'autres situations (par exemple, à l'unité des naissances, au département de l'urgence, à l'unité de soins intensifs) n'est pas abordé de manière spécifique dans le Guide, mais les mêmes principes devraient s'appliquer, quelle que soit la situation de transfert de patient. Le Guide lit désormais comme suit : «Le transfert peropératoire des soins entre deux anesthésiologistes doit être noté au dossier d'anesthésie et se conformer à un protocole structuré. » $\mathrm{La}$ communication des renseignements nécessaires au personnel de la salle de réveil devrait être effectuée «dans le cadre d'un transfert structuré du protocole de soins. »

À ce jour, le Guide n'a pas émis de recommandation quant à l'administration et au monitorage des bloqueurs neuromusculaires. Le Comité est un fier défenseur de l'optimisation de la sécurité des patients et, par conséquent, d'une attention rigoureuse en ce qui touche à l'utilisation de bloqueurs neuromusculaires non dépolarisants. Cette question est maintenant abordée dans la sous-section 5.3 Monitorage du patient - dans laquelle on peut lire : «Une posologie prudente, un monitorage vigilant et la neutralisation adéquate des bloqueurs neuromusculaires sont des éléments essentiels à la sécurité des patients. Un monitorage neuromusculaire devrait être utilisé lors de l'administration de bloqueurs neuromusculaires non dépolarisants. "

Les soins anesthésiques sont vulnérables et mis en péril pendant le transport des patients parce que le monitorage est temporairement interrompu. Par conséquent, le besoin d'un monitorage spécifique pendant le transport devrait être déterminé en fonction de la condition et du niveau de conscience du patient ainsi qu'en fonction du risque potentiel d'hypoxémie et de dépression respiratoire. Des dispositifs de monitorage adaptés devraient être utilisés lorsque indiqué, «tels que, entre autres, un saturomètre portable. » Un capnographe est requis pour les patients intubés ou sous sédation profonde (RSS 4-6) pendant leur séjour en salle de réveil. Cet énoncé avait été ajouté au Guide dans son édition révisée de $2016 .{ }^{6}$ Bien que la capnographie ne soit pas spécifiquement mentionnée pour le transport des patients dans le Guide, elle devrait être envisagée, lorsqu'elle est disponible, pendant le transport des patients intubés. Il faut parfois réintuber les patients présentant des voies aériennes difficiles en salle de réveil. Dans certains établissements, la salle de réveil est située à distance des salles d'opération, et il n'est pas toujours possible de transporter des patients en salle d'opération pour prendre en charge leurs voies aériennes. Ainsi, «Le matériel pour la prise en charge de voies aériennes difficiles doit être à portée immédiate en salle de réveil. "

Parmi les autres sujets qui font actuellement l'objet de discussions au Comité des normes quant à leur possible révision dans des éditions futures du Guide, citons des lignes directrices concernant la prise en charge de la douleur aiguë (par ex. les perfusions de kétamine), des recommandations pour la retraite et le 'ralentissement des 
activités' pré-retraite, ainsi que des directives spécifiques à l'anesthésie pédiatrique. Le Comité des normes continue de travailler en coopération avec le Comité sur les équipes de soin en anesthésie (CESA) afin d'appuyer l'élaboration continue du rôle de l'assistant en anesthésie et le guide de pratique reflété dans l'Annexe 5 : Exposé de principe sur les assistants en anesthésie. ${ }^{7} \mathrm{Au}$ nom du Comité des normes de la SCA, nous accueillons vos suggestions quant à d'autres questions et sujets qui devraient, selon vous, lecteurs, être abordés dans le présent Guide.

En conclusion, j'aimerais souligner le décès d'un des nôtres, une personne qui a joué un rôle prépondérant dans l'histoire de notre Guide. Ceux qui ont lu l'éditorial ${ }^{8}$ accompagnant l'édition révisée de 2017 et, en fait, bon nombre d'autres anesthésiologistes canadiens, savent que c'est le Dr John Feindel qui a créé la toute première version de notre Guide, publiée en 1977. Après une illustre carrière et une longue retraite, il est décédé en juillet 2017, à l'âge de 86 ans. Le Comité des normes tient à lui rendre hommage et à honorer sa vision, et nous offrons nos sincères condoléances à sa famille. Son héritage continuera de vivre dans notre Guide.

Conflicts of interest None declared.

Editorial responsibility This submission was handled by Dr. Hilary P. Grocott, Editor-in-Chief, Canadian Journal of Anesthesia.

\section{Conflit d'intérêt Aucun.}

Responsabilité éditoriale Cet article a été traité par Dr Hilary P. Grocott, rédacteur en chef, Journal canadien d'anesthésie.

\section{References}

1. Dobson G, Chong M, Chow L, et al. Guidelines to the Practice of Anesthesia - revised edition 2018. Can J Anesth 2018; 65. DOI:10. 1007/s12630-017-0995-9.

2. Canadian Anesthesiologists Society. Guidelines to the Practice of Anesthesia - Appendix 6. Position Paper on Procedural Sedation: An Official Position Paper of the Canadian Anesthesiologists' Society. Available from URL: https://www.cas.ca/English/ Guidelines (accessed September 2017).

3. Marshall $S$. The use of cognitive aids during emergencies in anesthesia: a review of the literature. Anesth Analg 2013; 117 : 1162-71.

4. Canadian Anesthesia Society. Choosing Wisely Canada Resources. Available from URL: https://www.cas.ca/English (accessed September 2017).

5. Mutter TC, Bryson GL. Choosing wisely and preoperative hemoglobin alc testing: what should it mean? Can J Anesth 2016; 63: 1307-13.

6. Merchant R, Chartrand D, Dain S, et al. Guidelines to the Practice of Anesthesia - revised edition 2016. Can J Anesth 2016; 63: 86112.

7. Canadian Anesthesiologists Society. Guidelines to the Practice of Anesthesia - Appendix 5. Position Paper on Anesthesia Assistants: An Official Position Paper of the Canadian Anesthesiologists' Society. Available from URL: https://www.cas.ca/English/ Guidelines (accessed September 2017).

8. Dobson G. Special announcement: Guidelines to the Practice of Anesthesia - revised edition 2017. Can J Anesth 2017; 64: 1-5. 